\title{
Resveratrol loaded polymeric micelles for theranostic targeting of breast cancer cells
}

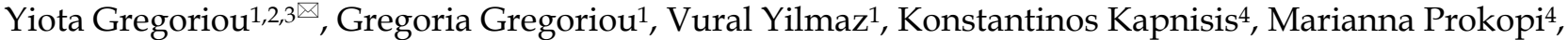 \\ Andreas Anayiotos ${ }^{4}$, Katerina Strati $^{1}$, Nikolas Dietis $^{5}$, Andreas I. Constantinou ${ }^{1}$ and Chrysafis Andreou ${ }^{2,3}$ \\ 1. Department of Biological Sciences, Faculty of Pure and Applied Sciences, University of Cyprus, Nicosia, Cyprus. \\ 2. Department of Electrical and Computer Engineering University of Cyprus, Nicosia, Cyprus. \\ 3. Emphasis Research Centre, University of Cyprus, Nicosia, Cyprus. \\ 4. Department of Mechanical Engineering and Material Science and Engineering, Cyprus University of Technology, Limassol, Cyprus. \\ 5. Medical School University of Cyprus, Nicosia, Cyprus. \\ $\triangle$ Corresponding authors: Yiota Gregoriou, Laboratory of Cancer Biology and Chemoprevention, Department of Biological Sciences, Faculty of Pure and Applied Sciences, \\ University of Cyprus, P.O. Box 20537, 1678 Nicosia, Cyprus. E-mail: gregoriou.panayiota@ucy.ac.cy; Chrysafis Andreou, Department of Electrical and Computer \\ Engineering, University of Cyprus, P.O. Box 20537, 1678 Nicosia, Cyprus. E-mail: andreou.chrysafis@ucy.ac.cy. \\ (C) The author(s). This is an open access article distributed under the terms of the Creative Commons Attribution License (https://creativecommons.org/licenses/by/4.0/). \\ See http://ivyspring.com/terms for full terms and conditions.
}

Received: 2020.08.14; Accepted: 2020.11.10; Published: 2021.01.01

\begin{abstract}
Treatment of breast cancer underwent extensive progress in recent years with molecularly targeted therapies. However, non-specific pharmaceutical approaches (chemotherapy) persist, inducing severe side-effects. Phytochemicals provide a promising alternative for breast cancer prevention and treatment. Specifically, resveratrol (res) is a plant-derived polyphenolic phytoalexin with potent biological activity but displays poor water solubility, limiting its clinical use. Here we have developed a strategy for delivering res using a newly synthesized nano-carrier with the potential for both diagnosis and treatment.

Methods: Res-loaded nanoparticles were synthesized by the emulsion method using Pluronic F127 block copolymer and Vitamin E-TPGS. Nanoparticle characterization was performed by SEM and tunable resistive pulse sensing. Encapsulation Efficiency (EE\%) and Drug Loading (DL\%) content were determined by analysis of the supernatant during synthesis. Nanoparticle uptake kinetics in breast cancer cell lines MCF-7 and MDA-MB-231 as well as in MCF-10A breast epithelial cells were evaluated by flow cytometry and the effects of res on cell viability via MTT assay.

Results: Res-loaded nanoparticles with spherical shape and a dominant size of $179 \pm 22 \mathrm{~nm}$ were produced. Res was loaded with high EE of $73 \pm 0.9 \%$ and DL content of $6.2 \pm 0.1 \%$. Flow cytometry revealed higher uptake efficiency in breast cancer cells compared to the control. An MTT assay showed that res-loaded nanoparticles reduced the viability of breast cancer cells with no effect on the control cells.

Conclusions: These results demonstrate that the newly synthesized nanoparticle is a good model for the encapsulation of hydrophobic drugs. Additionally, the nanoparticle delivers a natural compound and is highly effective and selective against breast cancer cells rendering this type of nanoparticle an excellent candidate for diagnosis and therapy of difficult to treat mammary malignancies.
\end{abstract}

Key words: cancer nanomedicine, nanotheranostics, resveratrol, breast cancer, drug-delivery

\section{Introduction}

Breast cancer therapy with molecularly targeted approaches has evolved significantly in the past few years, however, not all patients could benefit from such approach. As defined via gene expression analysis, breast cancer is not a homogeneous disease but rather a combination of unique and distinctive subtypes [1]. These subtypes are characterized by the expression of hormone receptors like the estrogen receptor (ER) and the progesterone receptor $(\mathrm{PgR})$, and the growth factor receptor HER2. This trio of cell-surface receptors are often exploited for the targeting of "magic bullet" drugs to the cancerous tissue. In the absence of these receptors targeting becomes very problematic and results in poor prognosis and treatment [2]. 
Triple negative breast cancer (TNBC) is a specific basal like subtype which is negative for all three markers - ER, PgR, and HER2 [3,4] and accounts for $15-20 \%$ of all breast cancer cases [5]. Due to TNBC's unique biology, hormonal and anti-HER2 therapies are ineffective, rendering this type of cancer clinically challenging. Additionally, TNBC is highly aggressive and highly metastatic [5]. Currently, the only available treatments for TNBC patients are limited to chemotherapy, radiation and surgery [6,7]. In current treatments, chemotherapy is the most employed choice. Although good chemotherapeutic agents exist, most fail to differentiate between healthy cells and malignant cells, resulting in systemic toxicity and severe side effects. It is therefore critical to develop more effective and less toxic strategies for the treatment of breast cancer.

Phytochemicals have been employed in breast cancer prevention and treatment with promising results [8]. Resveratrol (res), (trans-3,4',5-trihydroxystilbene, $\left.\mathrm{C}_{14} \mathrm{H}_{12} \mathrm{O}_{3}\right)$ is a plant-derived polyphenolic phytoalexin with potent biological activity. It is a non-flavonoid polyphenol found in abundancy in the skin of red grapes but has also been identified in other plant sources such as berries, soy beans, pomegranate and peanuts. Res has presented an array of medical benefits due to its interaction with a number of molecular targets [9-12]. For example, res found in red wine has been notoriously linked to the 'French paradox' for its cardioprotective effects. Besides its cardioprotective effects res has also been examined for its anticancer properties. Consequently, there is considerable evidence in the literature for the use of res as a chemopreventive and chemotherapeutic agent against various types of malignancies, including breast cancer [13-19]. Res's potential against mammary carcinogenesis has been well established in the literature and has been linked to various cell signaling pathways which cause cell cycle arrest, induce apoptosis, suppress proliferation, reduce inflammation and angiogenesis, and inhibit metastasis [20,13,15]. Furthermore, res behaves as a phytoestrogen and has been utilized in hormone-dependent therapy against ERa and PR subtypes of breast cancer [21]. Similarly, it has been shown that res enhances the cytotoxic efficacy of commonly used chemotherapeutic drugs [22] such as doxorubicin, in the treatment of a range of cancers [23]. Despite the abundance of literature supporting the use of res as a potent therapeutic agent, res exhibits poor clinical performance. This is most likely because res displays poor water solubility, short half-life and chemical instability in vivo. Consequently, achieving adequate bioavailability at an effective therapeutic dose in clinical studies has been the main impediment towards its clinical use [24-26].

Nano drug delivery systems have been utilized to circumvent the limitations associated with hydrophobic drug molecules like res [27]. Nanomaterialbased carriers offer a solution to problems associated with stability, solubility and toxicity of pharmaceuticals by offering protection from degradation, enabling controlled release and biodistribution and by increasing bioavailability through specific targeting. The development of biodegradable, polymer based nanocarriers, specifically ones prepared from amphiphilic block copolymers, has gained considerable attention in recent years. Polymeric micelles are a promising approach as they spontaneously self-assemble into nano-sized constructs. Normally, amphiphilic block copolymers composed of hydrophilic and hydrophobic segments can self-assemble into polymeric micelles at a concentration above their critical micelle concentration (CMC) [28-30].

In this work, we present an optimized method for preparing a nanodelivery system for breast cancer made from pluronic F127 block copolymer and using Vitamin E TPGS as the emulsifier for the delivery of res in vitro. This nanoparticle is unique because it comprises of a combination of biodegradable and biocompatible materials with known anticancer properties. Moreover, the same type of nanoparticle is also used to carry a fluorophore, Coumarin 6, offering the convenience of simultaneous diagnosis and therapy into a single nanoplatform [31-34].

Pluronic F127 is an amphiphilic triblock copolymer composed of poly (ethylene oxide) (PEO) and poly (propylene oxide) (PPO), PEO- $x$-PPO- $y$ PEO-x). Amphiphilic block copolymers self-assemble spontaneously in aqueous environments into polymeric nanostructures known as micelles and for this reason they have been commonly used to solubilize hydrophobic drugs in drug delivery. Shown schematically in Figure 1, the PEO block of F127 is hydrophilic and forms the outside layer of the nanoparticle while the PPO block is hydrophobic and composes the inner core. The hydrophobic core serves as a reservoir in which the hydrophobic drug molecule can be incorporated and protected from inactivation in biological media so it can be delivered effectively to the malignancy while the hydrophilic shell promotes the delivery of the drug to target cells $[35,28,36]$. Pluronic F127 has attracted a lot of attention in drug delivery because of its low toxicity in the body and the ability to encapsulate hydrophobic agents. Additionally, Pluronic F127 enhances pro-apoptotic signalling, thereby sensitizing tumour cells and making them more vulnerable to the effects of 
anticancer drugs $[31,33,34,37,38]$. Likewise, Vitamin E TPGS, a synthetic derivative of natural alphatocopherol, is an FDA and EFSA approved pharmaceutical adjuvant, frequently used in the development of DDS to improve the pharmacokinetics of anti-cancer drugs and reduce multi-drug resistance [39-43]. Additionally, Vitamin E TPGS has been shown to greatly enhance the performance of nanoparticles, resulting in much higher cellular uptake of the drug as well as more desirable in vivo pharmacokinetics [44].

In this study, the proposed nanoformulation was synthesized and subsequently characterized by particle size, morphology, encapsulation efficiency (EE) and drug loading (DL) content. Additionally, the formulation's performance as a diagnostic and therapeutic agent was evaluated by a cellular uptake assay and an in vitro cell viability assay using breast cancer cell lines MCF-7 and MDA-MB-231 as well as with immortalized MCF-10A breast epithelial cells. The nanoparticle showed an enhanced res EE of $73 \%$ and DL content of $6.2 \%$. Additionally, the nanoparticle showed superior uptake in breast cancer cells compared to control epithelial cells. Importantly, the nanoparticle reduced the viability of MCF-7 and MDA-MB-231 breast cancer cells with no effect on MCF-10A, rendering this nanoparticle a good candidate for diagnosis and therapy of difficult to treat mammary malignancies.

\section{Methods}

\section{Materials}

Res was purchased from Selleck chemicals (Dallas, TX., USA). The purity as determined by HPLC was $99.73 \%$. Pluronic F127 was purchased from Sigma-Aldrich (St. Louis, MO., U.S.A), coumarin 6 (MW 350.43) from Santa Cruz Biotechnology (Dallas, TX, USA) and D-Alpha Tocopheryl Polyethylene Glycol 1000 Succinate (Vitamin E TPGS) from Eastman Chemical Company (Kingsport, TN, USA). Acetone (ACS grade) and Dichloromethane anhydrous (purity $\geq 99.8 \%$ ) were purchased from Sigma-Aldrich and Dimethyl Sulfoxide, extra pure $(99.9 \%)$ from Scharlau chemicals (Barcelona, Spain).

\section{Preparation of the nanoparticle}

Res-loaded nanoparticles were prepared by the single-emulsion method. The preparation method comprises mixing Pluronic F127 $\left(\mathrm{EO}_{106} \mathrm{PO}_{70} \mathrm{EO}_{106}\right.$, $\mathrm{MW}=12,600)$ with dichloromethane as the solvent and D-alpha-tocopheryl polyethylene glycol 1000 succinate (Vitamin E TPGS) as the emulsifier. More specifically, $100 \mathrm{mg}$ of Pluronic F127 were mixed in 1 $\mathrm{mL}$ of dichloromethane and $50 \mu \mathrm{l}$ of res in acetone (at a concentration of $50 \mathrm{mg} / \mathrm{mL}$ ) were added to the polymer mixture by vortexing until the encapsulant was homogeneously dispersed. The polymer/ encapsulant solution was then added swiftly to Vitamin E TPGS at a ratio of 1:2 on high vortex. The resulting oil-in-water emulsion was ultrasonicated (Misonix, Ultrasonic Liquid Processors) in three $10 \mathrm{sec}$ bursts (50\% amplitude) on ice to induce nanosized droplets. The emulsion was then transferred into a beaker containing $45 \mathrm{~mL}$ of Vitamin E TPGS and stirred for $3 \mathrm{~h}$ to evaporate the solvent. The resulting hardened nanoparticles were then recovered by centrifugation on a fixed rotor at 17,000 rcf for $15 \mathrm{~min}$ at $22^{\circ} \mathrm{C}$ (Cientec CT-15000R centrifuge). The nanoparticles were washed 3 times via centrifugation with distilled water to remove any unentrapped drug. The supernatant containing unentrapped drug and unused polymer was collected and further tested to determine the drug entrapment efficiency of the nanoparticle. The resulting nanoparticle suspension was transferred to $15 \mathrm{~mL}$ pre-weighed falcon tubes, cooled to $-80^{\circ} \mathrm{C}$ for 3 hours and freeze-dried (Mecha Tech) for 48 hrs. For coumarin 6 loaded nanoparticles, coumarin 6 was added as the drug in place of res. Coumarin 6 was added directly to the polymer solution at a polymer to drug ratio of $1: 40$ by mass. The fabrication steps following were performed in the same manner as in the case of res-loaded nanoparticles and as mentioned above. The freeze-dried nanoparticles were wrapped in foil and stored at $4^{\circ} \mathrm{C}$ until further use. The freeze-dried nanoparticles were resuspended in ultrapure water (Sartorius Arium 611 VF Water Purifier system) and the solution was sonicated in a water bath sonicator (RS Pro, ultrasonic cleaner) three times for $15 \mathrm{~min}$ each time and subsequently filtered through a $0.45 \mu \mathrm{m}$ syringe filter (PTFE, Hydrophilic, Dissolution Accessories, (Oosterhout, The Netherlands)) to remove all aggregates and obtain nanoparticles of uniform size prior to use.

\section{Analysis of size and morphology}

\section{Scanning Electron Microscopy}

Morphological examination was performed with Scanning Electron Microscopy (SEM). Samples were mounted on aluminum specimen stubs and gold-sputtered to $5 \mathrm{~nm}$ thick films to prevent beam charging effects (SC7640 Sputter coater, Quorum Technologies, Kent, UK). High resolution scanning electron microscopic analysis was performed at $20 \mathrm{kV}$ (magnification range of 30,000-120,000×) using a FEI Quanta 200 (FEI, Oregon, USA) microscope and images were processed using the ImageJ software. 


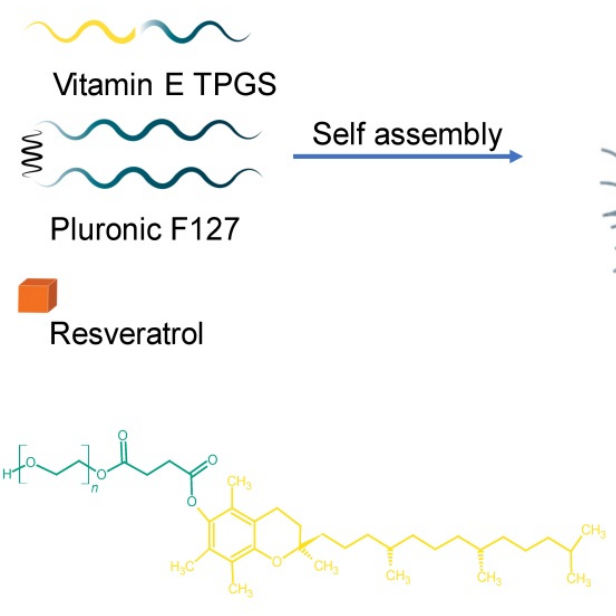

Vitamin E TPGS
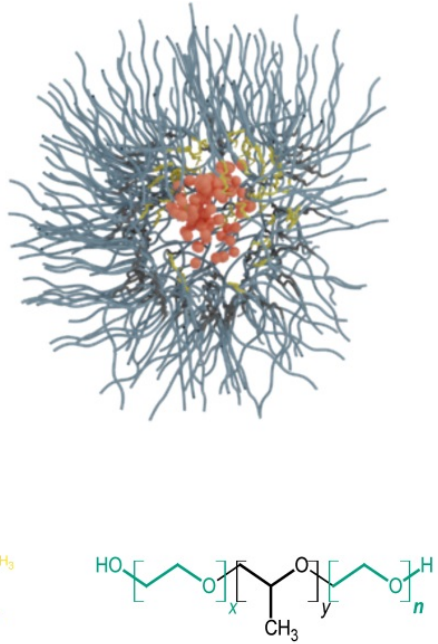

Pluronic F127

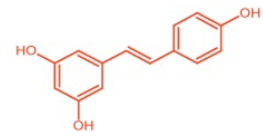

Trans Resveratrol

Figure 1. Schematic illustration for the preparation of resveratrol loaded nanoparticles. Hydrophilic regions for both Vitamin E TPGS and Pluronic F127 are represented by aqua blue and form the outside layer of the nanoparticle. The hydrophobic regions of Vitamin E TPGS and Pluronic F127 are coloured yellow and black, respectively and form the inner core. Resveratrol is encapsulated in the inner core and is illustrated by a brick colour. Chemical structures of $D$ - $\alpha$-tocopheryl polyethylene glycol 1000 succinate (Vitamin E TPGS), Pluronic F127 ( $x$ is 100 and $y$ is 65 ) and trans-Resveratrol are shown.
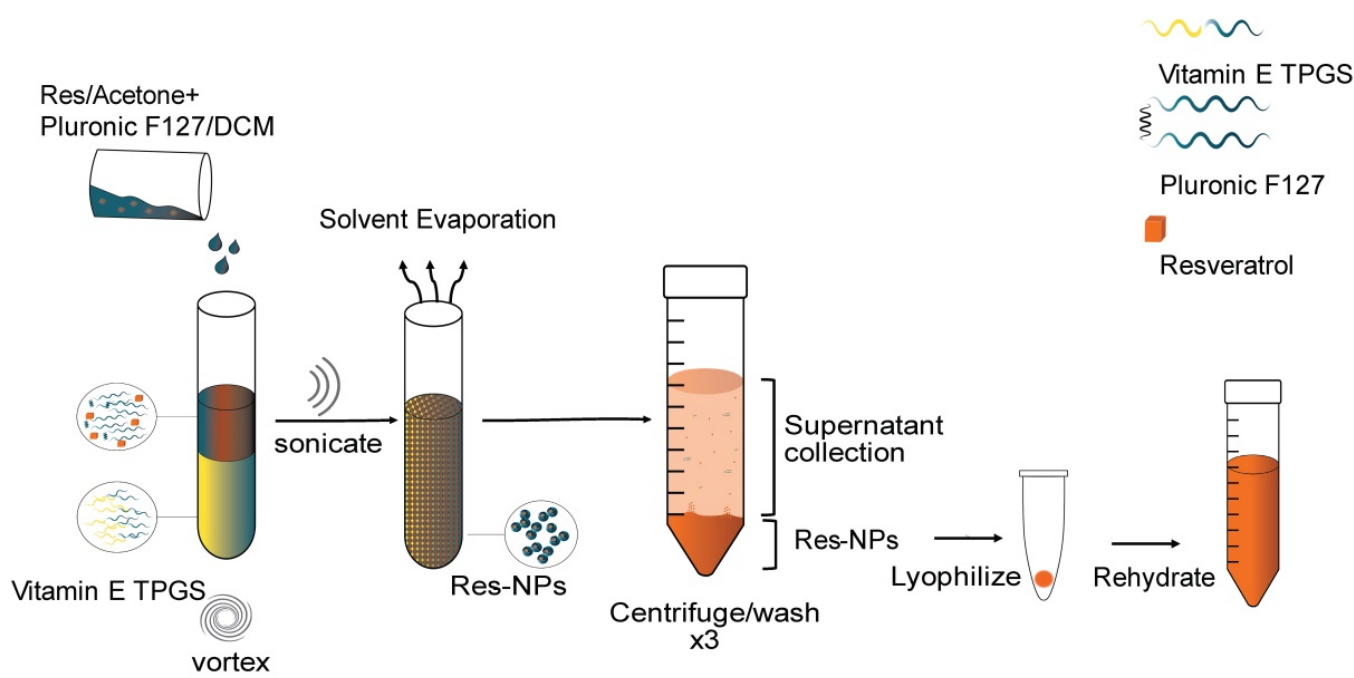

Figure 2. Schematic illustration for the synthesis of resveratrol (res) loaded nanoparticles. Res in acetone is mixed with dichloromethane (DCM) and then added to
Vitamin E TPGS under vortex. The resulting mixture is ultrasonicated and the solvent evaporated under stirring. The resulting hardened nanoparticles are centrifuged and washed with $\mathrm{d} . \mathrm{H} 2 \mathrm{O}$ three times to remove unentrapped drug, Vitamin E TPGS and Pluronic F127. The supernatant is collected for use in further characterization studies. The pellet is collected and lyophilized to obtain NPs in solid form. The lyophilized NPs are rehydrated before use.

\section{Tunable Resistive Pulse Sensing (TRPS) size analysis}

Quantification and size analysis of nanoparticles was performed using the qNano Gold platform (Izon Science, Oxford, U.K.). Nanoparticles were diluted in filtered PBS, measured using the nanopore NP200 (Izon Science, Oxford, U.K.) and compared to calibration particles CPC200. Data analysis was carried out using the Izon Control Suite software v3.3 (Izon Science, Oxford, U.K.).

\section{Composition analysis by UV-Vis}

The composition of the nanoparticles was verified by UV-Vis spectrophotometry (Perkin Elmer Lambda 1050, USA). For this purpose, the UV-Vis spectra of pure res, pure Pluronic F127, pure Vitamin
E TPGS, res-loaded-nanoparticles and empty nanoparticles were attained. The samples were scanned at a wavelength of $250-850 \mathrm{~nm}$. All solids and lyophilized nanoparticles were dissolved in DMSO prior to analysis.

\section{Determination of the Encapsulation Efficiency and Drug loading content}

The percent Encapsulation Efficiency (EE\%) and the percent Drug Loading (DL\%) content of res-loaded nanoparticles was determined by analysis of the supernatant obtained from the washes during synthesis (un-encapsulated drug) against a standard calibration curve of pure res. A representative $2 \mathrm{~mL}$ sample from each wash was centrifuged at 10,000 
r.p.m. for $10 \mathrm{~min}$ at room temperature. The supernatant was collected and analysed on a multimodal microplate reader (Tecan Spark 20M) to measure the fluorescence intensity of the sample (excitation wavelength of $356 \mathrm{~nm}$ and an emission wavelength of $383 \mathrm{~nm}$ ). For the calibration curve pure res was first diluted in DMSO to form a stock solution. This was further diluted in PBS to form a series of concentrations for the calibration curve. All experiments were performed in triplicates.

The \% entrapment efficiency (EE\%) is calculated from equation (1) [45]:

$$
\mathrm{EE} \%=\left(\mathrm{C}_{1} / \mathrm{C}_{2}\right) \times 100 \%
$$

The \% drug loading content (DL\%) is calculated from equation (2) [45]:

$$
\mathrm{DL} \%=\mathrm{C}_{1} /\left(\mathrm{C}_{2}+\mathrm{C}_{3}\right) \times 100 \%
$$

where $C_{1}$ is the amount of res encapsulated in res-loaded NPs, $C_{2}$ is the total amount of res used during synthesis and $C_{3}$ is the total amount of polymer used during synthesis.

\section{Cell culture and Reagents}

MCF-7, MDA-MB-231 and MCF-10A cell lines were obtained from the American Type Culture Collection (ATCC) (Manassas, VA). MCF-7 and MDA-MB-231 breast cancer cell lines were cultured in DMEM supplemented with $10 \%$ fetal bovine serum (FBS) and 1\% antibiotic/antimycotic. MCF-10A immortalized breast cell line was cultured in DMEM F12 supplemented with $20 \mathrm{ng} / \mathrm{mL}$ EGF, $100 \mathrm{ng} / \mathrm{mL}$ Cholera Toxin, $500 \mathrm{ng} / \mathrm{mL}$ Hydrocortizone, 10 $\mu \mathrm{g} / \mathrm{mL}$ Insulin, 5\% Horse Serum (HS) and 1\% antibiotic/antimycotic. Sub culturing of the cells was performed using $0.25 \%$ trypsin. The RPMI, FBS, antibiotics and trypsin used in cell culture were purchased from Gibco, Invitrogen (Carlsbad, California, USA).

\section{In vitro cellular uptake}

\section{Flow cytometry}

Considering that res is a non-fluorescent drug, coumarin 6 was incorporated as a fluorescent dye during nanoparticle formulation to study the cellular uptake of the nanoparticle in three cell lines, MCF-7, MDA-MB-231 and MCF-10A. MCF-7, MDA-MB-231 cell lines were seeded in 6 -well plates $\left(2 \times 10^{5} /\right.$ well $)$ and incubated with $250 \mu \mathrm{g} / \mathrm{mL}$ of coumarin 6-NP for 30, 60, 120 and 240 minutes. At the end of each incubation timepoint, cells were washed 3 times with PBS, harvested with trypsin and centrifuged for 5 minutes at $1100 \mathrm{rpm}$. Following supernatant aspiration, the cells were resuspended in $500 \mu \mathrm{L}$ of PBS prior FACs analysis. Data were acquired on a
Bio-Rad S3e Cell Sorter flow cytometer and analyzed using FlowJo software (Treestar). Representative FACS dot plots of gating strategy can be seen in the Figure S1. The population of cells was detected depending on their size and complexity (FSC-SSC gate) and later the doublets were excluded (FSC-Height/FSC-Area gate). Each experiment was performed in triplicate.

\section{Fluorescence microscopy}

To evaluate the cellular uptake of NPs in MCF-7, MDA-MB-231 and MCF-10A cell lines, coumarin 6, was used as a marker and was therefore encapsulated in the nanoparticles instead of res. Cells were seeded in 6-well plates at a density of $2 \times 10^{5}$ cells per well with coverslips and incubated overnight to allow for cell attachment. Cells were treated with $250 \mu \mathrm{g} / \mathrm{mL}$ of coumarin 6-NP and incubated for 30 and 240 minutes. At the end of the incubation, cells were washed 3 times with PBS, fixed with $4 \%$ paraformaldehyde (PFA) for 10 minutes. Cells were washed 4 times with PBS. To quench auto-fluorescence coverslips were incubated with $1 \mathrm{M}$ ammonium chloride for 15 minutes. Next, the coverslips were washed 3 times with PBS and mounted with DAPI mounting medium (Invitrogen/Thermo Fisher Scientific, Grand Island, NY USA). Fluorescence images were acquired on a Zeiss Axio Observer.A1 microscope. AxioVision and Photoshop CS6 software were used for image processing.

\section{MTT assay}

A total of $5 \times 10^{4}$ cells $/ \mathrm{mL}$ were seeded per well of a 96-well plate and incubated overnight to allow for cell attachment and recovery. At the end of each incubation period, cells were treated with different concentrations of res-loaded nanoparticles for the concentrations and time points described in the figure legends. Cell viability was measured using the MTT 3-(4,5-dimethylthiazol-2-yl)-2,5-monotetrazolium bromide assay. At the end of each incubation period, $20 \mu \mathrm{L}$ of MTT dye $(1 \mathrm{mg} / \mathrm{mL}$; Sigma St. Louis, MO) was added in each well and the plate was incubated at $37^{\circ} \mathrm{C}$ for 4 hours. Subsequently, the plates were read on a microplate reader (Wallac, PerkinElmer, Massachusetts, USA) at $570 \mathrm{~nm}$. Absorbance was proportional to the number of viable cells per well. Percentage of cell viability in each group was calculated after normalization to its own control. All data are presented as mean values \pm standard deviation and representative for at least two independent experiments performed in triplicates.

\section{Statistical analysis}

The data were expressed as the mean \pm standard deviation. $\mathrm{P}$ values were calculated by the one-way 
ANOVA test and at the 0.05 level were considered statistically significant. Data analysis was carried out by GraphPad Prism 8.0 (GraphPad Software Inc., San Diego, CA, USA) and OriginLab OriginalPro v. 8.5.1.

\section{Results and Discussion}

\section{Synthesis and characterization of NPs}

Res was encapsulated by Pluronic F127 to improve its water solubility and enhance its stability, thereby improving its pharmacological potential. Additionally, coumarin 6 was encapsulated in the same manner to serve for diagnostic purposes. Both nanoparticles were prepared by a modified single emulsion method [46] using dichloromethane (DCM) as the solvent and vitamin E TPGS as the emulsifying agent. Vitamin E TPGS is a superior stabilizing agent offering improved emulsification, superior encapsulation efficiency as well as rendering proapoptotic properties thereby adding to the anticancer capability of the nanoparticle. The resulting nanoparticle forms via the spontaneous self-assembly of Pluronic F127 at or above its CMC of around 0.1\% $(\mathrm{w} / \mathrm{v})$ [47-49]. Pluronic F127 is an interesting block copolymer for use as a carrier for cancer applications because of its known anticancer potential [31-34,38].

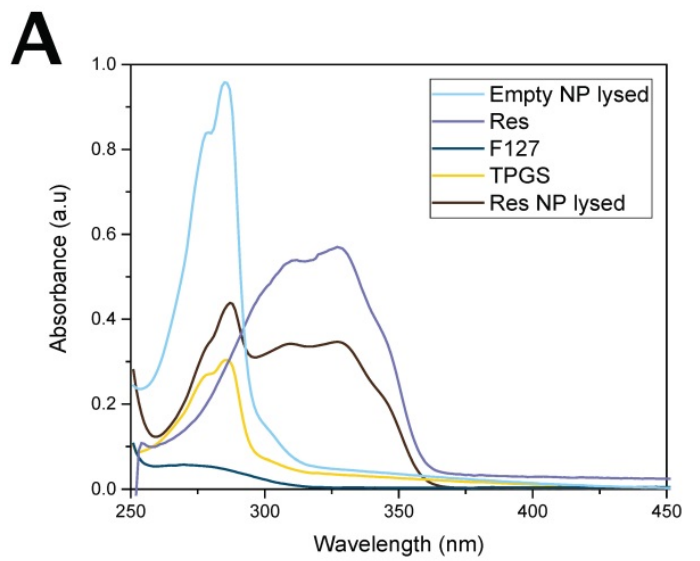

The resulting structure as illustrated in Figure 1 consists of a core-shell arrangement. The hydrophobic inner core carries the drug and involves the PPO hydrophobic segment of the polymer and the tocopherol segment of Vitamin E TPGS. The hydrophilic shell which surrounds the core is made of the hydrophilic PEO segment of the polymer and the PEG chains of Vitamin E TPGS. The hydrophilic shell renders the nanoparticle water soluble and enables the delivery of the hydrophobic drug in biological media. This carrier system is unique because all its constituents are non-toxic, biocompatible and biodegradable. Additionally, most of the constituents have shown promising anti-cancer properties. To the best of our knowledge this is the first time such a configuration of materials has been combined into a single platform.

The content of the nanoparticle was verified by UV-Absorption Spectroscopy. Figure 3A shows the UV spectra of Pluronic F127, res and Vitamin E TPGS as well as the spectra of res-loaded and empty nanoparticles. Res-loaded nanoparticles exhibit an absorbance peak at $318 \mathrm{~nm}$ characteristic of res which is absent in the empty nanoparticle spectrum confirming the presence of res in the nanoparticle.
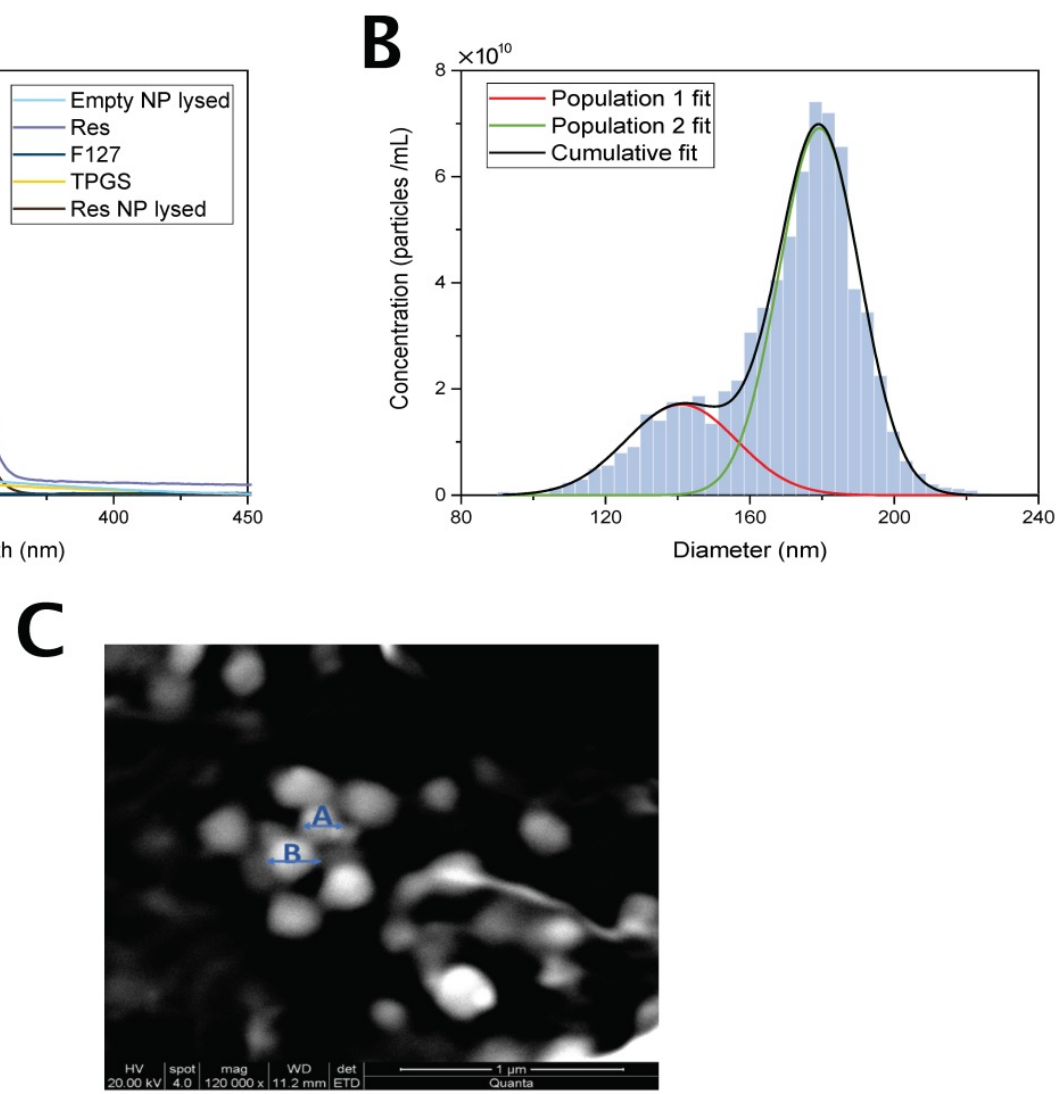

Figure 3. Nanoparticle characterization schematic. (A) UV-vis spectra of res, Pluronic F127, Vitamin E TPGS, Res-NP and Empty NP. Notes: Res shows a characteristic absorption peak at around $318 \mathrm{~nm}$. Abbreviations: Res, resveratrol; Res-NP, Resveratrol loaded nanoparticle; and Empty-NP, empty nanoparticle. (B) Particle size distribution obtained from TRPS analysis of res-loaded nanoparticles. Solid lines: Gaussian distribution fits of two nanoparticle populations detected (red and green) and cumulative particle size distribution (black). Abbreviations: Res-NP, Resveratrol loaded nanoparticle. (C) Scanning electron microscopy (SEM) images of res-loaded NPs. The scale bar is 1 um and the magnification during imaging was $120,000 \times$. A and B are two indicative particles with sizes of $140 \mathrm{~nm}$ and $179 \mathrm{~nm}$, respectively. 


\section{Nanoparticle characterization}

\section{Size analysis by TRPS}

The size and mean concentration of the nanoparticles were determined by TRPS and are shown in Figure 3B. Res-loaded nanoparticles appear to possess two size populations. The mean particle diameter of the most frequently occurring nanoparticles was $179 \pm 22 \mathrm{~nm}$ and that of the second frequently occurring was $140 \pm 30 \mathrm{~nm}$. The measured mean concentration was $7.24 \times 10^{10}$ particles $/ \mathrm{mL}$. The mean particle diameter of coumarin 6 loaded nanoparticles was $144 \pm 39 \mathrm{~nm}$ and the measured mean concentration was $2.73 \times 10^{10}$ particles $/ \mathrm{mL}$. The similarity in size between the two types of nanoparticles suggests that coumarin 6 was a good model reporter for res.

The dependency of nanoparticle size with nanoparticle physicochemical characteristics is well established in the literature [50-52]. Size determines the overall therapeutic efficacy of the nanoformulation by regulating nanoparticle biodistribution and tumor penetration as well as cellular internalization. Moreover, size governs nanoparticle clearance from the blood and excretion from body. Over the past few decades a consensus has been reached in regards to the desired size for cancer applications. If only in view of size variances nanoparticles ranging from $100 \mathrm{~nm}$ to $200 \mathrm{~nm}$ are preferred due to their extended blood circulation and the relatively slow rate of uptake by the reticuloendothelial system (RES) allowing for enhanced therapeutics [53]. Consequently, most clinically approved nanoformulations for cancer applications have sizes in the range of 100 to $200 \mathrm{~nm}[54,55]$. The particle sizes in both res-loaded and coumarin 6-loaded nanoformulations are between $100 \mathrm{~nm}$ and $200 \mathrm{~nm}$ rendering these newly synthesized nanoparticles suitable for drug delivery.

\section{Morphology and size analysis by SEM}

The morphology of res loaded nanoparticles was examined by SEM. As shown in Figure $3 \mathrm{C}$ the nanoparticles are homogeneous and exhibit a spherical shape. The size as determined by SEM was estimated to be $184.42 \pm 38 \mathrm{~nm}$.

\section{Determination of the Encapsulation Efficiency and Drug loading content}

The entrapment efficiency was found to be $73 \pm 0.9 \%$ and the drug loading content was $6.2 \pm 0.1 \%$. The entrapment efficiency and the drug loading content are important parameters to consider when accessing the nanomedicine's therapeutic effect and function. The entrapment efficiency is indicative of the extent of encapsulation of the drug in feed during the fabrication process and informs of the success of the entrapment [56]. This parameter is mainly related to the preparation method of the nanomedicine and the amount of drug added during synthesis. Although the mechanism of entrapment was not investigated in this work, this high EE value could be attributed to the hydrophobicity of res and the interactions between res and the hydrophobic PPO segment of the polymer as well as with the aromatic ring of Vitamin E TPGS inside the core of the nanoparticle [57]. The drug loading content on the other hand mirrors the ratio of drug to the entire nanoconstruct, demonstrating the percentage of mass of the nanoparticle that is owed to the encapsulated drug [45]. The DL content is highly dependent on the physical and chemical properties of the carrier polymer. Generally speaking a drug loading content of less than $10 \%$ is considered low [58-60].

\section{Characterization of nanoparticle uptake in breast cancer cell lines using coumarin 6 as a model drug}

\section{Cellular Uptake by Flow Cytometry}

Quantitative analysis of nanoparticle uptake kinetics of fluorescent nanoparticles was evaluated by flow cytometry and the results are presented in Figure 4. Coumarin 6 was used as the fluorescent model drug. The concentration of nanoparticle used was calculated taking the whole nanoparticle weight into account and corresponds to approximately 2.5 $\mu \mathrm{g} / \mathrm{mL}$ of drug. Three cell lines, MDA-MB-231, MCF-7 and MCF-10A were incubated with the nanoparticles and their fluorescence was quantified by flow cytometry at various time points up to $4 \mathrm{~h}$ of incubation. To investigate the nanoformulations' differential uptake in transformed vs. non-transformed cells we used two invasive breast carcinoma cell lines (MDA-MB-231 and MCF-7) and non-malignant breast epithelial cells (MCF-10A). Both types are invasive breast carcinoma cells but possess various phenotypic/genotypic differences. Mainly, MCF-7 are hormone dependent while MDA-MB-231 are triple negative. The quantitative analysis by flow cytometry was very promising indicating that nanoparticle uptake in breast cancer cells was more effective compared to that in normal cells - with the TNBC cell line, MDA-MB-231, showing the most dramatic uptake. The Box-Lucas function, $y=Y_{o}\left(1-\mathrm{e}^{-b x}\right)$ was used to fit the data, where $y$ is the Mean Fluorescence Intensity (MFI), $x$ is time (min), $Y_{0}$ is the maximum MFI (a.u) and $b=1 / \tau$. More specifically,

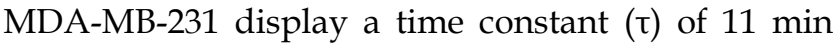
and a $Y_{0}$ of 27,300 (a.u), MCF-7 display a time constant of $46 \mathrm{~min}$ and a $Y_{0}$ of 9,618 (a.u) and MCF-10A display 
a time constant of $140 \mathrm{~min}$ and a $\mathrm{Y}_{0}$ of 8,748 (a.u). These results suggest specificity against cancer cells and the potential to reduce side effects if administered in vivo. Although the mechanism of entry was not examined in this study, a possible explanation for this difference in nanoparticle uptake between cell lines could be attributed to the ability of Pluronic block copolymers to induce changes in the microviscosity of cell membranes [38,61]. Melik-Nubarov et al. [38] reported that these changes in the microviscosity are thought to occur as a result of the adsorption of the block copolymers on the cell membrane which consequently induced alterations in the membrane's structure. Most importantly, the alterations in membrane structure varied depending on whether the cell was cancerous or not. In fact, the effect was opposite with certain Pluronics increasing the microviscosity of cancerous cells but decreasing the microviscosity ('solidified') of the membrane of normal cells. Additionally, membrane fluidization in cancerous cells causes inhibition of the Pgp efflux function [62]. This effect is exaggerated in our case due to the presence of Vitamin E TPGS which is known to do the same. Consequently, the enhanced uptake kinetics in MDA-MB-231 cells compared to MCF-7 could be explained due to higher Pgp expression in this type of cancer cell line [63]. The enhanced nanoparticle internalization capacity seen in MDA-MB-231 cells is promising considering the limited treatment options for this specific type of cancer.

A

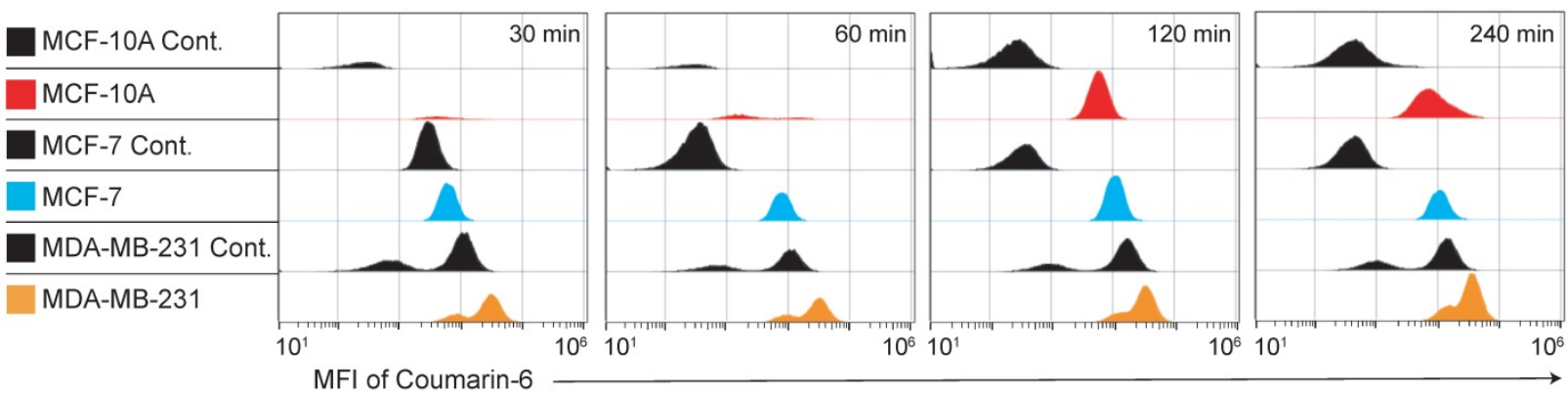

B

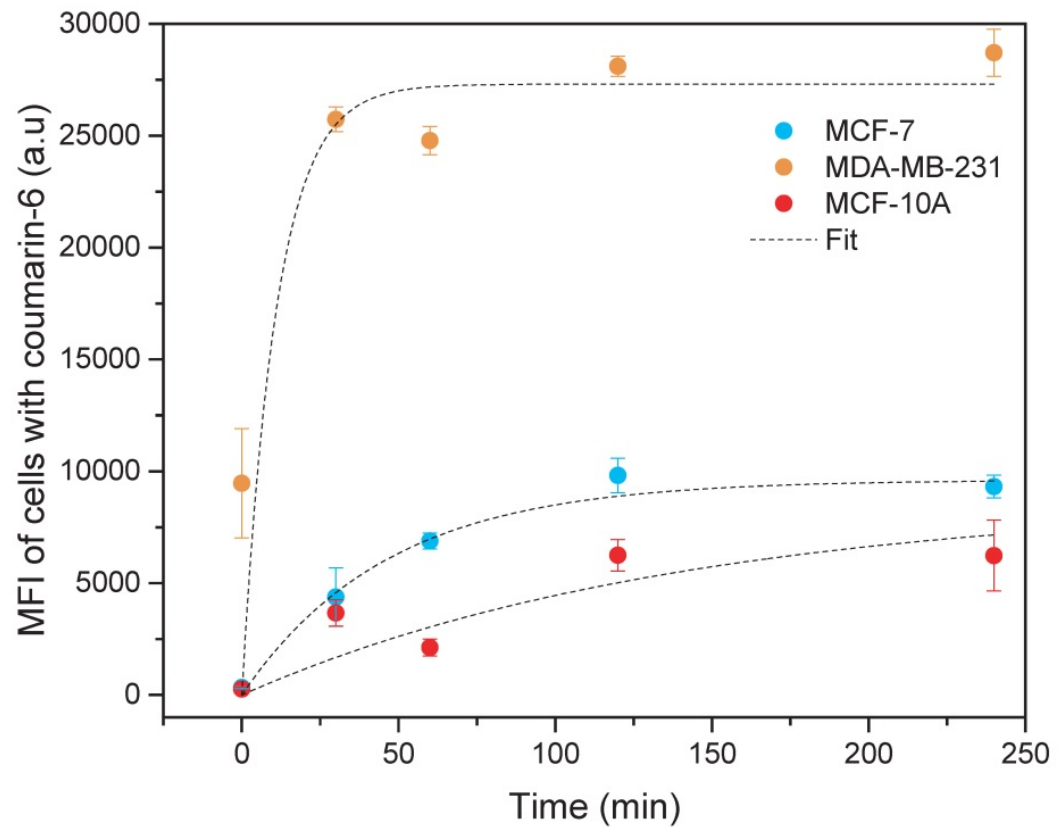

Figure 4. Time dependent cellular uptake analysis by flow cytometry. MDA-MB-231, MCF-7 and MCF-10A cells were incubated with Coumarin-6 loaded nanoparticles for up to $4 \mathrm{~h}$. (A) Histograms of all cell lines at the given treatment groups showing the MFI of coumarin 6; MCF-7 cells (blue), MCF-10A (red) and MDA-MB-231 (orange). All controls are shown in black (B) Time kinetics of nanoparticle uptake in MDA-MB-231, MCF-7 and MCF-10A cell lines. Results are represented as mean MFI value \pm SD, $n=3$. Data were analyzed by Origin Pro and fitted with Box-Lucas function Abbreviation: Mean Fluorescence Intensity (MFI). 


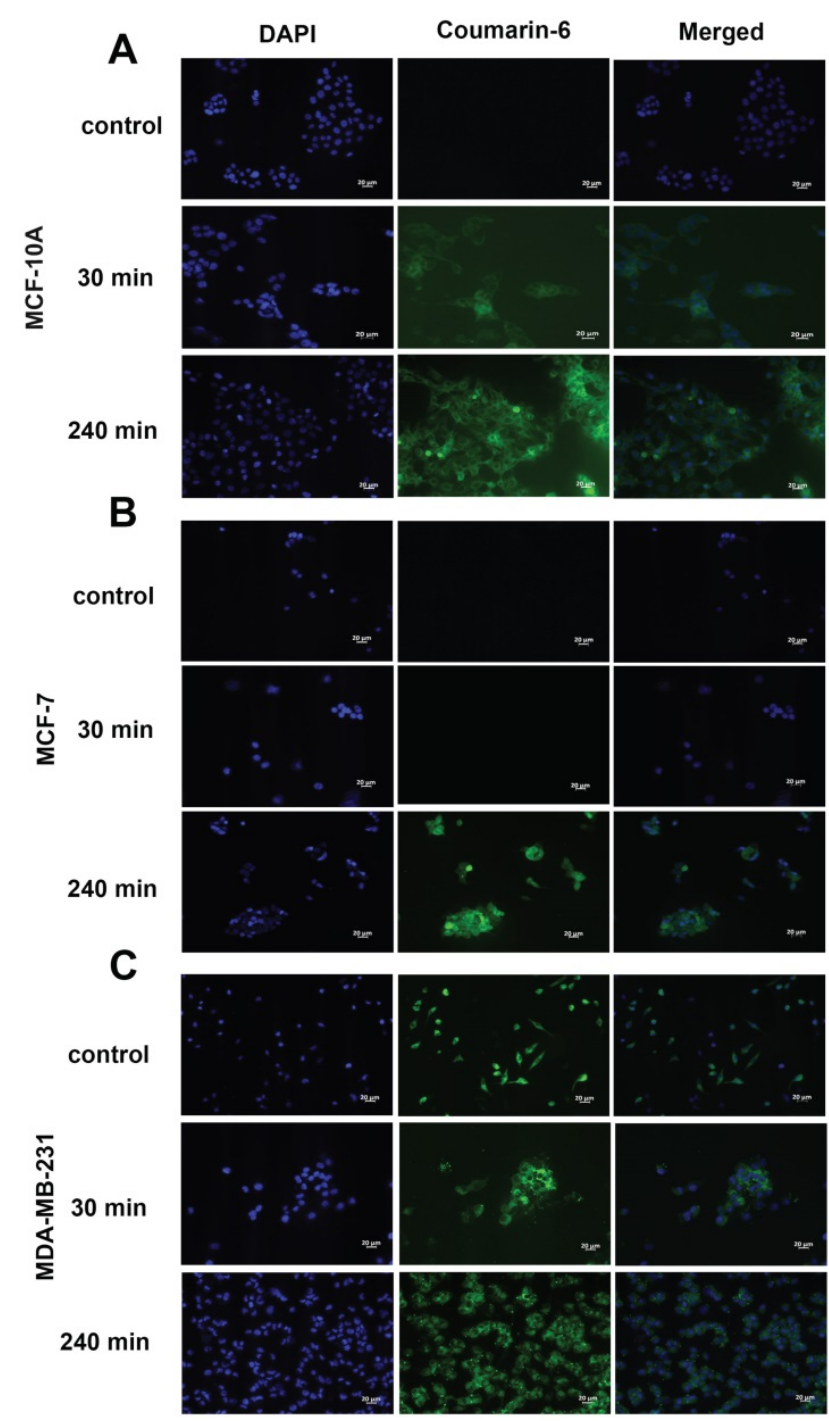

Figure 5. Fluorescence microscopy of cells treated with coumarin-6 loaded nanoparticles. In all panels blue represents nuclear staining (DAPI) and green represents coumarin-6 at 20x magnification. MCF-10A, MCF-7 and MDA-MB-231 cells were treated with $250 \mu \mathrm{g} / \mathrm{mL}$ of coumarin $6 \mathrm{NPs}$ for 30 minutes and 240 minutes. Green fluorescence was observed in the cytoplasm around the nuclei of cells, suggesting uptake of coumarin 6 loaded NPs. Scale bar: $20 \mu \mathrm{m}$.

\section{In vitro cellular uptake}

To qualitatively assess the uptake of nanoparticles by MCF-10A, MCF-7 and MDA-MB-231 cell lines, fluorescence microscopy was used. The cells were incubated with the nanoparticles loaded with coumarin 6 for $30 \mathrm{~min}$ and $240 \mathrm{~min}$. The fluorescent images, as seen in Figure 5, clearly indicate nanoparticles are taken up into the cytoplasm of all cell lines at $4 \mathrm{~h}$. Uptake was not seen at $30 \mathrm{~min}$ in MCF-7 (Fig. 5B) cells, contrary to the trend seen in flow cytometry. Additionally, morphological changes were observed in MDA-MB-231 cells upon treatment with res-loaded NPs. The MDA-MB-231 cell line changed from a spindle shaped morphology as seen in the control to a more spherical morphology at 30 and $240 \mathrm{~min}$ (Fig. 5C).

\section{Selective decrease in cell viability of breast cancer cell lines by res-loaded nanoparticles}

MCF-7, MDA-MB-231 breast cancer and MCF-10A immortalized breast cell lines were treated with different concentrations of res-loaded nanoparticles (as indicated in Figure 6) for 24, 48 and $72 \mathrm{~h}$. The concentrations presented were calculated based only on the amount of res encapsulated in the nanoparticle. As shown in Figure 6, res-loaded nanoparticles were more effective and specific in reducing cell proliferation of MDA-MB-231 and MCF-7 cancer cell lines, and most importantly, with no significant effects on the 'normal' MCF-10A cell line. Specifically, at concentrations ranging from $0.5-2.5 \mu \mathrm{g} / \mathrm{mL}$, res-loaded nanoparticles induced a marked reduction on cell viability of MDA-MB-231 and MCF-7 cell lines. Specifically, at a concentration of $2.5 \mu \mathrm{g} / \mathrm{mL}$, MDA-MB-231 cell viability dropped to $39.8 \%$ at $24 \mathrm{~h}, 14.0 \%$ at $48 \mathrm{~h}$ and $7.1 \%$ at $72 \mathrm{~h}$, while the MCF-7 cell viability reduced to $58.7 \%$ at $24 \mathrm{~h}$, $37.9 \%$ at $48 \mathrm{~h}$ and $20.7 \%$ at $72 \mathrm{~h}$. The $\mathrm{IC}_{50}$ values are shown in Table 1. On the contrary, we did not observe any significant effects on the MCF-10A cell line at all concentrations and timepoints tested, which agrees with the previous results observed using flow cytometry. The $p$ values can be seen in Table S1.

Table 1. IC50 values of res-loaded nanoparticles for all cell-lines at $24 \mathrm{~h}, \mathbf{4 8} \mathrm{h}$ and $72 \mathrm{~h}$. IC 50 values were calculated using the GraphPad Prism 8.0 (GraphPad Software Inc., San Diego, CA, USA). N/A is used in cases where cell viability did not reach $50 \%$

\begin{tabular}{llll}
\hline $\mathrm{IC}_{50}$ & & & \\
\hline & $24 \mathrm{~h}$ & $48 \mathrm{~h}$ & $72 \mathrm{~h}$ \\
\hline MDA-MB-231 & $2.32 \pm 0.12$ & $1.06 \pm 0.12$ & $0.76 \pm 0.04$ \\
MCF-7 & N/A & $1.61 \pm 0.57$ & $0.93 \pm 0.09$ \\
MCF-10A & N/A & N/A & N/A \\
\hline
\end{tabular}

\section{Conclusion}

In this study, we have successfully prepared and characterized a drug delivery system made from Pluronic F127 and Vitamin E TPGS for the encapsulation of res and coumarin 6 , producing a fully soluble drug formulation. The resulting nanoparticle was effective at selectively targeting aggressive forms of breast cancer with no significant uptake by immortalized healthy epithelial cells. Additionally, the nanoparticle showed higher reduction in cell viability of breast cancer cells with no significant toxic effect on immortalized breast cells. These results suggest that the proposed nanoparticle is a promising platform for delivering drugs to breast cancer cells with a dual purpose: diagnosis and treatment. 

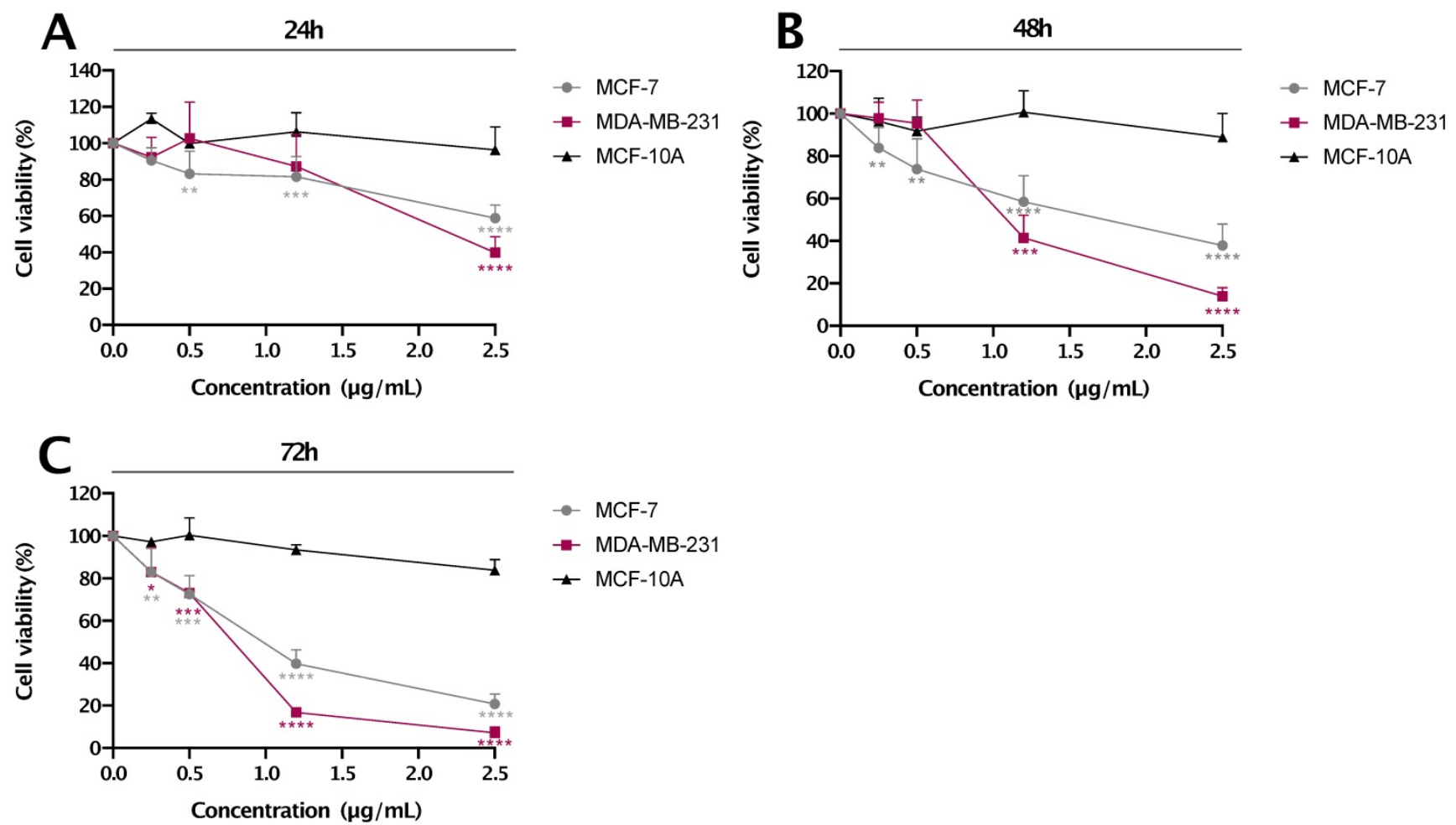

Figure 6. The $\%$ cell viability vs. concentration $(\mu \mathrm{g} / \mathrm{mL}$ ) plot for res-loaded nanoparticles. The anti-proliferative effect of res-loaded nanoparticles on MCF-10A, MCF-7 and MDA-MB-231 cell lines was examined at concentrations and timepoints as indicated: (A) treated for $24 \mathrm{~h}$, (B) treated for $48 \mathrm{~h}$ and (C) treated for $72 \mathrm{~h}$. All data are presented as mean values \pm standard deviation and are representative of at least two independent experiments performed in triplicate. $P$ values: $*<0.05, * *<0.01$, $* * *<0.001$, $* * * *<0.0001$ relative to control.

\section{Abbreviations}

CMC: critical micelle concentration; DCM: dichloromethane; DL: Drug Loading; EE: Encapsulation Efficiency; ER: estrogen receptor; FBS: fetal bovine serum; MFI: Mean Fluorescence Intensity; MTT: 3-(4,5-dimethylthiazol-2-yl)-2,5-diphenyltetrazolium bromide; PEO: poly (ethylene oxide); PFA: paraformaldehyde; PgR: progesterone receptor; $\mathrm{PPO}$ : poly (propylene oxide); SEM: scanning electron microscopy; TNBC: triple negative breast cancer; TRPS: tunable resistive pulse sensing; Vitamin E TPGS: D-alpha-tocopheryl polyethylene glycol 1000 succinate.

\section{Supplementary Material}

Supplementary figures and tables.

http://www.ntno.org/v05p0113s1.pdf

\section{Acknowledgements}

We would like to thank Dr. David T. Cramb at Ryerson University for his help with attaining funding, Dr. Paris Papageorgis for his help with data analysis and Mrs. Panayiota Marcou for her help with fluorescence imaging.

\section{Funding}

This work was supported by funding from the University of Cyprus and was co-funded by the European Regional Development Fund and the Republic of Cyprus through the Research and Innovation Foundation (Projects: INTERNATIONAL/OTHER/0118/0017, OPPORTUNITY/ 0916/ERC-StG/003， INFRASTRUCTURES/1216/ 0034, POST-DOC/0916/0111, and INTERNATIONAL/OTHER/0118/0018).

\section{Contributions}

YG attained funding for this work, designed, coordinated and supervised all experiments performed, performed all experiments related to nanoparticle synthesis and characterization, performed the fluorescence microscopy, analyzed results and drafted the manuscript. GG performed the cell cultures, the MTT assays, flow cytometry, fluorescence microscopy, data analysis and assisted with the writing of the manuscript. VY performed the flow cytometry and analyzed the results from this assay. MP performed the qNano analysis. CK performed the SEM. AA, KS and ND provided support in data interpretation. AIC proposed the idea to make res TPGS nanoparticles. AIC and CA 
provided help with data analysis, interpretation and editing of the manuscript.

\section{Competing Interests}

The authors have declared that no competing interest exists.

\section{References}

1. Perou CM, Sørlie T, Eisen MB, van de Rijn M, Jeffrey SS, Rees CA, et al. Molecular portraits of human breast tumours. Nature. 2000; 406: 747-52.

2. Dawood S. Triple-negative breast cancer: epidemiology and management options. Drugs. 2010; 70: 2247-58.

3. Sørlie T, Perou CM, Tibshirani R, Aas T, Geisler S, Johnsen H, et al. Gene expression patterns of breast carcinomas distinguish tumor subclasses with clinical implications. Proceedings of the National Academy of Sciences of the United States of America. 2001; 98: 10869-74

4. Sorlie T, Tibshirani R, Parker J, Hastie T, Marron JS, Nobel A, et al. Repeated observation of breast tumor subtypes in independent gene expression data sets. Proceedings of the National Academy of Sciences of the United States of America. 2003; 100: 8418-23.

5. Anders CK, Carey LA. Biology, Metastatic Patterns, and Treatment of Patients with Triple-Negative Breast Cancer. Clinical Breast Cancer. 2009; 9: S73-S81.

6. Kutanzi KR, Yurchenko OV, Beland FA, Checkhun VF, Pogribny IP. MicroRNA-mediated drug resistance in breast cancer. Clin Epigenetics. 2011; 2: $171-85$

7. Willers H, Würschmidt F, Janik I, Bünemann H, Heilmann HP. [Combined breast-preserving surgery, chemotherapy and radiotherapy in the treatment of breast carcinoma. Effect of the interval between surgery and the beginning of radiotherapy]. Strahlentherapie und Onkologie: Organ der Deutschen Rontgengesellschaft [et al]. 1997; 173: 148-54.

8. Bonofiglio D, Giordano C, De Amicis F, Lanzino M, Andò S. Natural Products as Promising Antitumoral Agents in Breast Cancer: Mechanisms of Action and Molecular Targets. Mini reviews in medicinal chemistry. 2016; 16: 596-604.

9. Catalgol B, Batirel S, Taga Y, Ozer NK. Resveratrol: French paradox revisited. Frontiers in pharmacology. 2012; 3: 141.

10. Mobasheri A, Shakibaei M. Osteogenic effects of resveratrol in vitro: potential for the prevention and treatment of osteoporosis. Annals of the New York Academy of Sciences. 2013; 1290: 59-66.

11. Pasinetti GM, Wang J, Ho L, Zhao W, Dubner L. Roles of resveratrol and other grape-derived polyphenols in Alzheimer's disease prevention and treatment. Biochimica et Biophysica Acta (BBA) - Molecular Basis of Disease. 2015; 1852: 1202-8.

12. Borriello A, Bencivenga D, Caldarelli I, Tramontano A, Borgia A, Zappia V, et al. Resveratrol: from basic studies to bedside. Cancer treatment and research. 2014; 159: 167-84.

13. Aggarwal BB, Bhardwaj A, Aggarwal RS, Seeram NP, Shishodia S, Takada Y. Role of resveratrol in prevention and therapy of cancer: preclinical and clinical studies. Anticancer research. 2004; 24: 2783-840.

14. Pezzuto JM. Resveratrol as an Inhibitor of Carcinogenesis. Pharmaceutical Biology. 2008; 46: 443-573.

15. Bishayee A. Cancer prevention and treatment with resveratrol: from rodent studies to clinical trials. Cancer prevention research (Philadelphia, Pa). 2009; 2: 409-18.

16. Bishayee A, Politis T, Darvesh AS. Resveratrol in the chemoprevention and treatment of hepatocellular carcinoma. Cancer treatment reviews. 2010; 36: 43-53.

17. Carter LG, D'Orazio JA, Pearson KJ. Resveratrol and cancer: focus on in vivo evidence. Endocr Relat Cancer. 2014; 21: R209-R25.

18. Benitez DA, Pozo-Guisado E, Alvarez-Barrientos A, Fernandez-Salguero PM, Castellón EA. Mechanisms involved in resveratrol-induced apoptosis and cell cycle arrest in prostate cancer-derived cell lines. Journal of andrology. 2007; 28: $282-93$

19. Cao Y, Fu ZD, Wang F, Liu HY, Han R. Anti-angiogenic activity of resveratrol, a natural compound from medicinal plants. Journal of Asian natural products research. 2005; 7: 205-13.

20. Sinha D, Sarkar N, Biswas J, Bishayee A. Resveratrol for breast cancer prevention and therapy: Preclinical evidence and molecular mechanisms. Seminars in Cancer Biology. 2016; 40-41: 209-32.

21. Mehdawi $\mathrm{H}$, Alkhalaf $\mathrm{M}$, Khan I. Role of $\mathrm{Na}+\mathrm{H}+$ exchanger in resveratrolinduced growth inhibition of human breast cancer cells. Medical oncology (Northwood, London, England). 2012; 29: 25-32.

22. Ito $\mathrm{Y}$, Mitani $\mathrm{T}$, Harada $\mathrm{N}$, Isayama $\mathrm{A}$, Tanimori $\mathrm{S}$, Takenaka $\mathrm{S}$, et al. Identification of carbonyl reductase 1 as a resveratrol-binding protein by affinity chromatography using 4'-amino-3,5-dihydroxy-trans-stilbene. Journal of nutritional science and vitaminology. 2013; 59: 358-64.

23. Al-Abd AM, Mahmoud AM, El-Sherbiny GA, El-Moselhy MA, Nofal SM, El-Latif HA, et al. Resveratrol enhances the cytotoxic profile of docetaxel and doxorubicin in solid tumour cell lines in vitro. Cell Prolif. 2011; 44: 591-601.
24. Smoliga JM, Blanchard O. Enhancing the delivery of resveratrol in humans: if low bioavailability is the problem, what is the solution? Molecules (Basel, Switzerland). 2014; 19: 17154-72.

25. Pangeni R, Sahni JK, Ali J, Sharma S, Baboota S. Resveratrol: review on therapeutic potential and recent advances in drug delivery. Expert opinion on drug delivery. 2014; 11: 1285-98

26. Smoliga JM, Vang O, Baur JA. Challenges of translating basic research into therapeutics: resveratrol as an example. The journals of gerontology Series A, Biological sciences and medical sciences. 2012; 67: 158-67.

27. Santos AC, Veiga F, Ribeiro AJ. New delivery systems to improve the bioavailability of resveratrol. Expert opinion on drug delivery. 2011; 8: 973-90.

28. Kumari A, Yadav SK, Yadav SC. Biodegradable polymeric nanoparticles based drug delivery systems. Colloids and surfaces B, Biointerfaces. 2010; 75: 1-18.

29. Masood F. Polymeric nanoparticles for targeted drug delivery system for cancer therapy. Materials science \& engineering C, Materials for biological applications. 2016; 60: 569-78.

30. Neophytou CM, Gregoriou Y, Constantinou AI. Pro-apoptotic Properties of Chemopreventive Agents. In: Pezzuto JM, Vang O, editors. Natural Products for Cancer Chemoprevention: Single Compounds and Combinations. Cham: Springer International Publishing. 2020; p:517-59.

31. Alakhov V, Kabanov A. Block Copolymer-Based Formulations of Doxorubicin Effective Against Drug Resistant Tumours. In: Chiellini E, Sunamoto J, Migliaresi C, Ottenbrite RM, Cohn D, editors. Biomedical Polymers and Polymer Therapeutics. Boston, MA: Springer US. 2002; p: 121-37.

32. Kabanov AV, Batrakova EV, Alakhov VY. Pluronic block copolymers as novel polymer therapeutics for drug and gene delivery. Journal of controlled release: official journal of the Controlled Release Society. 2002; 82: 189-212.

33. Alakhov V, Moskaleva E, Batrakova EV, Kabanov AV. Hypersensitization of multidrug resistant human ovarian carcinoma cells by pluronic P85 block copolymer. Bioconjugate chemistry. 1996; 7: 209-16.

34. Batrakova EV, Li S, Brynskikh AM, Sharma AK, Li Y, Boska M, et al. Effects of pluronic and doxorubicin on drug uptake, cellular metabolism, apoptosis and tumor inhibition in animal models of MDR cancers. Journal of controlled release: official journal of the Controlled Release Society. 2010; 143: 290-301.

35. Kazunori K, Glenn S K, Masayuki Y, Teruo O, Yasuhisa S. Block copolymer micelles as vehicles for drug delivery. Journal of Controlled Release. 1993; 24: 119-32.

36. Imran M, Shah M, Ullah S. Amphiphilic block copolymers based micelles for drug delivery. 2017

37. Kabanov AV, Batrakova EV, Alakhov VY. Pluronic ${ }^{\circledR}$ block copolymers as novel polymer therapeutics for drug and gene delivery. Journal of Controlled Release. 2002; 82: 189-212.

38. Melik-Nubarov NS, Pomaz OO, Dorodnych T, Badun GA, Ksenofontov AL, Schemchukova OB, et al. Interaction of tumor and normal blood cells with ethylene oxide and propylene oxide block copolymers. FEBS letters. 1999; 446: 194-8.

39. Katz J, Blake E, Medrano TA, Sun Y, Shiverick KT. Isoflavones and gamma irradiation inhibit cell growth in human salivary gland cells. Cancer letters. 2008: 270: 87-94

40. Schmidt F, Knobbe CB, Frank B, Wolburg H, Weller M. The topoisomerase II inhibitor, genistein, induces $\mathrm{G} 2 / \mathrm{M}$ arrest and apoptosis in human malignant glioma cell lines. Oncology reports. 2008; 19: 1061-6.

41. Chodon D, Ramamurty N, Sakthisekaran D. Preliminary studies on induction of apoptosis by genistein on HepG2 cell line. Toxicology in vitro: an international journal published in association with BIBRA. 2007; 21: 887-91.

42. Neophytou CM, Constantinou C, Papageorgis P, Constantinou AI. D-alpha-tocopheryl polyethylene glycol succinate (TPGS) induces cell cycle arrest and apoptosis selectively in Survivin-overexpressing breast cancer cells. Biochemical pharmacology. 2014; 89: 31-42.

43. Neophytou CM, Constantinou AI. Drug Delivery Innovations for Enhancing the Anticancer Potential of Vitamin E Isoforms and Their Derivatives. BioMed research international. 2015; 2015: 584862.

44. Neophytou CM, Constantinou AI. Drug Delivery Innovations for Enhancing the Anticancer Potential of Vitamin E Isoforms and Their Derivatives. BioMed research international. 2015; 2015. 584862

45. Carissimi G, et al. Direct Quantification of Drug Loading Content in Polymeric Nanoparticles by Infrared Spectroscopy. Pharmaceutics. 2020; 12.

46. McCall RL, Sirianni RW. PLGA nanoparticles formed by single- or double-emulsion with vitamin E-TPGS. Journal of visualized experiments : JoVE. 2013; p: 51015.

47. Sharma PK, Bhatia SR. Effect of anti-inflammatories on Pluronic ${ }^{\circledR}$ F127: micellar assembly, gelation and partitioning. International Journal of Pharmaceutics. 2004; 278: 361-77.

48. Escobar-Chávez JJ, López-Cervantes M, Naïk A, Kalia YN, QuintanarGuerrero D, Ganem-Quintanar A. Applications of thermo-reversible pluronic F-127 gels in pharmaceutical formulations. Journal of pharmacy \& pharmaceutical sciences: a publication of the Canadian Society for Pharmaceutical Sciences, Societe canadienne des sciences pharmaceutiques. 2006; 9: 339-58.

49. Kabanov A, Lemieux P, Vinogradov S, Alakhov V. Pluronic block copolymers: novel functional molecules for gene therapy $\mathrm{a}, \mathrm{b}$ a b. Advanced drug delivery reviews. 2002; 54: 223-33.

50. Peer D, Karp JM, Hong S, Farokhzad OC, Margalit R, Langer R. Nanocarriers as an emerging platform for cancer therapy. Nature Nanotechnology. 2007; 2: $751-60$ 
51. Clancy AA, Gregoriou Y, Yaehne K, Cramb DT. Measuring properties of nanoparticles in embryonic blood vessels: Towards a physicochemical basis for nanotoxicity. Chemical Physics Letters. 2010; p: 99-111.

52. Yaehne K, Tekrony A, Clancy A, Gregoriou Y, Walker J, Dean K, et al. Nanoparticle accumulation in angiogenic tissues: towards predictable pharmacokinetics. Small (Weinheim an der Bergstrasse, Germany). 2013; 9: 3118-27.

53. Moghimi SM, Hunter AC, Andresen TL. Factors controlling nanoparticle pharmacokinetics: an integrated analysis and perspective. Annual review of pharmacology and toxicology. 2012; 52: 481-503.

54. Uster PS, Working PK, Vaage J. Pegylated liposomal doxorubicin (DOXIL®, CAELYX®) distribution in tumour models observed with confocal laser scanning microscopy. International Journal of Pharmaceutics. 1998; 162: 77-86.

55. Gradishar WJ, Tjulandin S, Davidson N, Shaw H, Desai N, Bhar P, et al. Phase III trial of nanoparticle albumin-bound paclitaxel compared with polyethylated castor oil-based paclitaxel in women with breast cancer. Journal of clinical oncology : official journal of the American Society of Clinical Oncology. 2005; 23: 7794-803.

56. Shen S, Wu Y, Liu Y, Wu D. High drug-loading nanomedicines: progress, current status, and prospects. International journal of nanomedicine. 2017; 12: 4085-109.

57. Gao Y, Li LB, Zhai G. Preparation and characterization of Pluronic/TPGS mixed micelles for solubilization of camptothecin. Colloids and surfaces B, Biointerfaces. 2008; 64: 194-9.

58. Zheng S, Xie Y, Li Y, Li L, Tian N, Zhu W, et al. Development of high drug-loading nanomicelles targeting steroids to the brain. International journal of nanomedicine. 2014; 9: 55-66.

59. Qin SY, Zhang AQ, Cheng SX, Rong L, Zhang XZ. Drug self-delivery systems for cancer therapy. Biomaterials. 2017; 112: 234-47.

60. Cai K, He X, Song Z, Yin Q, Zhang Y, Uckun FM, et al. Dimeric Drug Polymeric Nanoparticles with Exceptionally High Drug Loading and Quantitative Loading Efficiency. Journal of the American Chemical Society. 2015; 137: 3458-61.

61. Batrakova EV, Li S, Vinogradov SV, Alakhov VY, Miller DW, Kabanov AV. Mechanism of pluronic effect on P-glycoprotein efflux system in blood-brain barrier: contributions of energy depletion and membrane fluidization. The Journal of pharmacology and experimental therapeutics. 2001; 299: 483-93.

62. Regev R, Assaraf YG, Eytan GD. Membrane fluidization by ether, other anesthetics, and certain agents abolishes P-glycoprotein ATPase activity and modulates efflux from multidrug-resistant cells. European journal of biochemistry. 1999; 259: 18-24.

63. Chen J, Lu L, Feng Y, Wang H, Dai L, Li Y, et al. PKD2 mediates multi-drug resistance in breast cancer cells through modulation of P-glycoprotein expression. Cancer letters. 2011; 300: 48-56. 